\title{
Local SAR compression with overestimation control to reduce maximum relative SAR overestimation and improve multi-channel RF array performance
}

\author{
Stephan Orzada ${ }^{1,2}$ (]) $\cdot$ Thomas M. Fiedler ${ }^{3} \cdot$ Andreas K. Bitz $^{4} \cdot$ Mark E. Ladd $^{1,3,5,6} \cdot$ Harald H. Quick $^{1,2}$
}

Received: 23 April 2020 / Revised: 31 August 2020 / Accepted: 11 September 2020 / Published online: 22 September 2020

(c) The Author(s) 2020

\begin{abstract}
Purpose In local SAR compression algorithms, the overestimation is generally not linearly dependent on actual local SAR. This can lead to large relative overestimation at low actual SAR values, unnecessarily constraining transmit array performance.

Method Two strategies are proposed to reduce maximum relative overestimation for a given number of VOPs. The first strategy uses an overestimation matrix that roughly approximates actual local SAR; the second strategy uses a small set of pre-calculated VOPs as the overestimation term for the compression.

Result Comparison with a previous method shows that for a given maximum relative overestimation the number of VOPs can be reduced by around $20 \%$ at the cost of a higher absolute overestimation at high actual local SAR values.

Conclusion The proposed strategies outperform a previously published strategy and can improve the SAR compression where maximum relative overestimation constrains the performance of parallel transmission.
\end{abstract}

Keywords SAR $\cdot$ Vops $\cdot$ VOP compression $\cdot$ MRI $\cdot$ Local SAR

\section{Introduction}

Stephan Orzada

Stephan.orzada@uni-due.de

1 Erwin L. Hahn Institute for Magnetic Resonance Imaging, University Duisburg-Essen, Kokereiallee 7, 45141 Essen, Germany

2 High-Field and Hybrid MR Imaging, University Hospital Essen, 45147 Essen, Germany

3 Medical Physics in Radiology, German Cancer Research Center (DKFZ), Im Neuenheimer Feld 280, 69120 Heidelberg, Germany

4 Electromagnetic Theory and Applied Mathematics, Faculty of Electrical Engineering and Information Technology, FH Aachen, University of Applied Sciences, 52066 Aachen, Germany

5 Faculty of Physics and Astronomy, University of Heidelberg, Im Neuenheimer Feld 226, 69120 Heidelberg, Germany

6 Faculty of Medicine, University of Heidelberg, Im Neuenheimer Feld 672, 69120 Heidelberg, Germany
While single-channel and dual-channel transmit systems are still standard in clinical MRI systems, multi-channel parallel transmit ( $\mathrm{pTx}$ ) radiofrequency $(\mathrm{RF})$ systems are often used at ultra-high field (UHF). Not only are these systems necessary to cope with the inhomogeneity introduced by the short wavelength of the RF signals [1,2], these systems also offer more flexibility in excitation, especially at UHF $[3,4]$. Examples of the techniques utilizing pTx systems are RF shimming [5, 6], kT-points [7], 2D spokes [8], 3D tailored radiofrequency pulses [9], Transmit SENSE [10, 11], and TIAMO [12]. Common among all these techniques is the use of arbitrary amplitudes and phases on the different transmit channels (excitation vector), whereby they differ in how rapidly the vectors are changed over time.

Altering the excitation vector changes the field patterns generated by the transmit system through its coil array including the E-fields in addition to the H-fields, and, therefore, the distribution of RF power absorption in the body tissue is changed as well. Regulatory guidelines [13] recommend constraints on specific absorption rate (SAR) averaged globally over the whole body (or, when appropriate, 
the region of the body exposed to RF fields), and averaged locally over any $10 \mathrm{~g}$ of tissue.

For a given combination of coil and subject on a singlechannel system there is a fixed ratio between global SAR $\left(\mathrm{SAR}_{\text {global }}\right)$ and maximum local SAR $\left(\mathrm{SAR}_{\text {max,local }}\right)$, whereas in a pTx system there is no direct relation between the two. Furthermore, while in a single-channel system there is a direct relationship between input power and SAR, both $\mathrm{SAR}_{\text {global }}$ and $\mathrm{SAR}_{\text {local }}$ depend on the complex values of the excitation vector in a pTx system.

To ensure safety in pTx systems, numerical simulations are performed that use models of the arrays together with heterogeneous body models to calculate the fields inside the tissue [14]. These simulations provide maps of the local SAR that can be used to determine global and $10 \mathrm{~g}$ averaged SAR. This information can then be used for pulse design with SAR constraints as well as for online SAR supervision [15-17], but the number of voxels from such a simulation is very high and can reach orders of $10^{6}$.

To reduce the complexity of SAR calculation, the concept of virtual observation points (VOPs) [18] was introduced to compress the number of matrices. The general idea is to trade the number of voxels against an overestimation of the actual local SAR. Instead of calculating the SAR over all voxels, only a reduced number of VOPs need to be calculated. With this concept, the number of calculations necessary to approximate $\mathrm{SAR}_{\text {max,local }}$ can be reduced by several orders of magnitude. While the original clustering algorithm of Eichfelder et al. [18] is quite commonly used, a greedy algorithm was presented by Lee et al. [19] that achieves an even lower number of VOPs for a given problem and overestimation factor.

A drawback of both concepts is the fact that the overestimation is not (or at least not directly) dependent on $\mathrm{SAR}_{\text {max,local. }}$. In the case of the Eichfelder algorithm, it is dependent on the worst-case SAR $_{\text {max,local }}$ multiplied by a scalar In the case of Lee, it is dependent on $\mathrm{SAR}_{\text {global }}$ multiplied by a scalar. This implies that the maximum absolute overestimation over all excitation vectors with unit power is fixed in relation to actual maximum local SAR for the Eichfelder algorithm and only slightly varying for the Lee algorithm. However, since the difference between the worstcase $\mathrm{SAR}_{\text {max,local }}$ and the lowest $\mathrm{SAR}_{\text {max,local }}$ can be more than an order of magnitude, the maximum relative overestimation can be significant. In practice this may lead to an unnecessary reduction of the duty cycle by a factor that is equivalent to the relative overestimation or a reduction of the allowed flip angle proportional to the square root of the relative overestimation.

For a typical array the worst-case $\mathrm{SAR}_{\max , \text { local }}$ for a fixed input power is much higher than the lowest possible $\mathrm{SAR}_{\text {max,local }}$ for that same power, so the maximum relative overestimation is likely to be high, thus unnecessarily constraining array performance. This problem can be expected to be even more pronounced for arrays with a large numbers of coil elements, which can be illustrated by a simple example. In a close-fitting coil array, high local SAR can occur especially beneath the individual elements [20]. When all the power of a unit power excitation vector is transmitted via a single element, high local SAR will occur at this position, while a uniform distribution of power to all channels will lead to lower local SAR at least directly beneath the elements. The higher the number of channels, the more the power will be concentrated or distributed, respectively.

In this work, we present two ways of reducing maximum relative overestimation. The two compression schemes are both based on the greedy algorithm presented by Lee et al. [19]. The first one uses a matrix that roughly approximates the local SAR instead of using the global SAR matrix. The second one uses a set of VOPs to generate the necessary overestimation. These two new schemes are compared against Lee's method.

\section{Materials and methods}

\section{Overestimation with global SAR Matrix ("S $S_{\text {gobal }}$ ")}

Lee et al. define their VOP condition for the complete set $V_{\text {all }}$ of $10 \mathrm{~g}$-averaged SAR matrices $S_{v, 10 \mathrm{~g}}$ by

$\max _{v \in V_{\text {all }}}\left\{b^{\prime} S_{v, 10 \mathrm{~g}} b\right\} \leq \max _{w \in V_{\text {sub }}}\left\{b^{\prime} S_{w, 10 \mathrm{~g}} b\right\}+\varepsilon_{\mathrm{G}} b^{\prime} S_{\text {Global }} b \forall b \in \mathbb{C}^{N_{\text {channel }}}$

Here $b$ is the excitation vector, $N_{\text {channel }}$ is the number of channels, $V_{\text {sub }} \subseteq V_{\text {all }}$ is a subset of the SAR matrices, $S_{w, 10 \mathrm{~g}}$ are the SAR matrices from the subset $V_{\text {sub }}, \varepsilon_{\mathrm{G}}$ is the overestimation factor, and $S_{\text {Global }}$ is the global SAR matrix that calculates the global SAR from the input vector. We will call this strategy " $S_{\text {global }}$ " in the following parts of the manuscript, and it will serve as a reference standard for the two new algorithms. The rightmost term, $\varepsilon_{\mathrm{G}} b^{\prime} S_{\mathrm{Global}} b$, we call the overestimation term. In this work, we propose different strategies that use different overestimation terms.

\section{Overestimation with a constant factor (" $S_{\text {diag }}$ ")}

Another strategy would be to use a constant overestimation term that is independent of the actual SAR. This can be achieved by replacing $S_{\text {Global }}$ in Eq. (1) by a matrix $S_{\text {diag }}$ that is a diagonal matrix with all elements of the main diagonal having the same value while all other values are zero. This is equivalent to using the spectral norm in Eq. (6) of Eichfelder's paper. It has some similarity with the commonly used approach of using the Frobenius norm in Eq. (6) of said paper in the sense that the overestimation is independent of 
the actual SAR. The main difference is that due to the difference in the algorithms, Eichfelder's iterative algorithm when using the Frobenius norm only adds overestimation in dimensions where it is necessary, while Lee's algorithm adds the overestimation in all directions. The most notable difference being that the worst case SAR of an Eichfelder VOP set is the same the worst case SAR of the uncompressed set of SAR matrices, while the worst case SAR of a Lee VOP set is the worst case SAR of the uncompressed set of SAR matrices plus overestimation. In the following parts of the manuscript, we will call this strategy " $S_{\text {diag }}$ ", and it will serve as the second reference standard for the two new algorithms.

\section{Overestimation with approximated local SAR ("S local")}

Since the relation between $S_{\text {Global }}$ and local SAR cannot be expressed by means of a constant proportionality factor, the overestimation term in Eq. (1) only places an upper bound on the absolute overestimation error, but not the relative overestimation, which can lead to very large relative errors. To control the maximum relative overestimation, we propose using a matrix $S_{\text {local }}$ that is a rough approximation of local SAR:

$\max _{v \in V_{\text {all }}}\left\{b^{\prime} S_{v, 10 \mathrm{~g}} b\right\} \leq \max _{w \in V_{\text {sub }}}\left\{b^{\prime} S_{w, 10 \mathrm{~g}} b\right\}+\varepsilon_{\mathrm{G}} b^{\prime} S_{\text {local }} b$

Adding up all SAR matrices with appropriate weighting results in a matrix $S_{\text {Global }}$ that provides global SAR as described by Lee et al. [19], for example. To calculate a matrix $S_{\text {local }}$ that represents local SAR better, the weighting has to be changed. A simple approach for this is to calculate a VOP set and add up the VOPs, thereby putting more weight on those matrices that give a good representation of local SAR.

To calculate $S_{\text {local }}$ we use the original algorithm proposed by Lee to determine a subset $V_{\text {sub,pre }} \subsetneq V_{\text {all, } 1 \%} \subsetneq V_{\text {all }}$, where we reduce the complexity of the calculation by first randomly selecting a subset of only $1 \%$ of all SAR matrices $\left(V_{\text {all, } 1 \%}\right)$ to which we apply the Lee algorithm. We then sum up the matrices of the calculated sub volume $V_{\text {sub,pre }}$ (VOPs) to get a matrix $S_{\text {pre }}$ that has some properties of local SAR.

$S_{\text {pre }}=\sum_{v \in V_{\text {sub.pre }}} S_{v, 10 \mathrm{~g}}$

To achieve an even better approximation of local SAR, we calculate the eigenvectors of $S_{\text {pre }}$ and calculate the local SAR for these eigenvectors from the full set of SAR matrices. $S_{\text {local }}$ is then defined as follows:

$S_{\text {local }}=V D V^{\prime}$, where $V$ is a matrix whose columns are the eigenvectors and $D$ is a diagonal matrix where the diagonal element $d_{i i}$ is the SAR value $\mathrm{SAR}_{\text {maxlocal eig } \mathrm{i}}$ corresponding to the $i$ th eigenvector. Since the difference between the highest and the lowest SAR value in $D$ can be quite large and lead to extreme differences in the overestimation, we suggest using an exponential scaling term $R \geq 1$ to reduce the ratio and empirically find a matrix that provides good results (low maximum relative overestimation) in the compression:

$d_{i i}=\mathrm{SAR}_{\text {maxlocal eig } \mathrm{i}}^{\frac{1}{R}}$

We suggest to use an $R$ so that the ratio between the maximum and the minimum eigenvalue of $S_{\text {local }}$ is below 10 .

In the following parts of the manuscript, we will call this strategy " $S_{\text {local }}$ ", and it is one of the two new algorithms we propose to control maximum relative overestimation.

\section{Overestimation by pre calculated VOPs ("Double VOP")}

While the above approach of using a single overestimation matrix to approximate local SAR for the overestimation term is simple in that after calculating $S_{\text {local }}$ the algorithm as defined in [19] can be used, it has the drawback that $S_{\text {local }}$ only provides a very rough correlation with local SAR. To get a better correlation, we can use the maximum local SAR value calculated from a small pre-calculated set of VOPs $V_{\text {sub,pre }}$ for the overestimation:

$\max _{v \in V_{\text {all }}}\left\{b^{\prime} S_{v, 10 \mathrm{~g}} b\right\} \leq \max _{w \in V_{\text {sub }}}\left\{b^{\prime} S_{w, 10 \mathrm{~g}} b\right\}+\varepsilon_{\mathrm{G}} \max _{u \in V_{\text {sub,pre }}}\left\{b^{\prime} S_{u, 10 \mathrm{~g}} b+\varepsilon_{\mathrm{G}, \mathrm{pre}} b^{\prime} S_{\mathrm{Global}} b\right\}$

Here $\varepsilon_{\mathrm{G}, \text { pre }}$ is the overestimation factor used to calculate the set of pre-calculated VOPs $V_{\text {sub,pre }}$. This set can be calculated by using the algorithm proposed by Lee et al. The scaling factor $\varepsilon_{\mathrm{G}}$ can be used to scale the maximum overestimation.

At first, it might appear that Eq. (6) is similar to

$\max _{v \in V_{\text {all }}}\left\{b^{\prime} S_{v, 10 \mathrm{~g}} b\right\} \leq\left(1+\varepsilon_{\mathrm{G}}\right) \max _{w \in V_{\text {sub }}}\left\{b^{\prime} S_{w, 10 \mathrm{~g}} b\right\}$,

i.e., using a simple scaling factor, but during compression these two equations are obviously not equivalent. By looking at the very first step of the greedy algorithm, this becomes clear. In the first step the matrix with the highest eigenvalue is compared to the matrix with the second highest eigenvalue. In the case of Eq. (6) a term is added containing information on the maximum local SAR over all voxels (plus an overestimation term); in the case of Eq. (7) only the information from the very first matrix is used. Over the course of the algorithm, this leads to many more voxels being included in the subset. 
When applying Eq. (6), the algorithm proposed by Lee et al. [19] has to be modified slightly. First we replace the condition in Eq. (6) by its identical condition in terms of matrix inequalities [21, 22], equivalent to how it was done in [19]:

$S_{v, 10 \mathrm{~g}} \leq \sum_{w \in V_{\text {sub }}} c_{w, v} S_{w, 10 \mathrm{~g}}+\varepsilon_{\mathrm{G}} \sum_{u \in V_{\mathrm{pre}}} c_{u, v}\left(S_{u, 10 \mathrm{~g}}+\varepsilon_{\mathrm{G}, \mathrm{pre}} S_{\mathrm{Global}}\right)$

In each greedy step of the algorithm, the iterative method from Lee et al. [19] is used, with modifications necessary to incorporate the changed terms:

1. Initialization of the two sets of coefficients $c_{w, v}$ and $c_{u, v}$. In each set of coefficients, the coefficients are set to equal values with an L1 norm of 1 .

2. If all the eigenvalues of the matrix $P=\sum_{w \in V_{\text {sub }}} c_{w, v} S_{w, 10 \mathrm{~g}}+\varepsilon_{\mathrm{G}} \sum_{u \in V_{\text {pre }}} c_{u, v}\left(S_{u, 10 \mathrm{~g}}+\varepsilon_{\mathrm{G}, \mathrm{pre}} S_{\mathrm{Global}}\right)-S_{v, 10 \mathrm{~g}}$ are nonnegative, the voxel $v$ can be upper-bounded by the previously determined VOPs.

3. If not, calculate the eigenvector $b$ of $P$ corresponding to the minimum eigenvalue of $P$. If $b^{\prime} S_{v, 10 \mathrm{~g}} b>\max _{w \in V_{\text {sub }}}$ $\left\{b^{\prime} S_{w, 10 \mathrm{~g}} b\right\}+\varepsilon_{\mathrm{G}} \max _{u \in V_{\text {pre }}}\left\{b^{\prime} S_{u, 10 \mathrm{~g}} b+\varepsilon_{\mathrm{G}, \mathrm{pre}} S_{\mathrm{Global}}\right\}$, the voxel $v$ cannot be upper-bounded and is included in the subset.

4. If neither the condition from step 2 nor from step 3 is satisfied, update the coefficients to make $b^{\prime} \mathrm{Pb}$ nonnegative:

5. Calculate $m=b^{\prime} \mathrm{Pb}$

6. a. Randomly change one coefficient of $c_{w, v}$ and normalize so that the $\mathrm{L} 1$ norm remains 1 . Calculate $m_{\mathrm{temp}}=b^{\prime} \mathrm{Pb}$.
If $m_{\text {temp }}$ is nonnegative, go to step 5. If $m_{\text {temp }}$ is smaller than $m$, undo the coefficient change, else set $m=m_{\text {temp }}$

7. b. Randomly change one coefficient of $c_{u, v}$ and normalize so that the $\mathrm{L} 1$ norm remains 1 and calculate $m_{\text {temp }}=b^{\prime} \mathrm{Pb}$. If $m_{\text {temp }}$ is nonnegative, go to step 5. If $m_{\text {temp }}$ is smaller than $m$, undo the coefficient change, else set $m=m_{\text {temp }}$

8. c. Repeat

9. Iterate step $2-4$. If any of the conditions are not satisfied by the time the number of iterations exceeds a preselected maximum, add the voxel to $V_{\text {sub }}$.

This algorithm results in two sets of VOPs. The maximum of the pre-calculated set is multiplied by $\varepsilon_{\mathrm{G}}$ and added to each SAR value of the newly calculated set, then these values can be used just like the results of the other algorithms. In the following parts of the manuscript, we will call this strategy "Double VOP", and it is the second of the two new algorithms we propose to control maximum relative overestimation.

\section{Coil arrays}

To test the algorithms, the SAR matrices of two different eight-channel arrays made from micro strip lines with meanders [23] and operating at the proton resonance frequency of $7 \mathrm{~T}$ were used. The first array is a local array placed directly on the body [24], while the second array is a remote array in $2 \times 4$ configuration positioned behind the bore liner (Fig. 1 ). All simulations were performed in CST Microwave Studio 2017 (CST AG, Darmstadt, Germany) and considered the
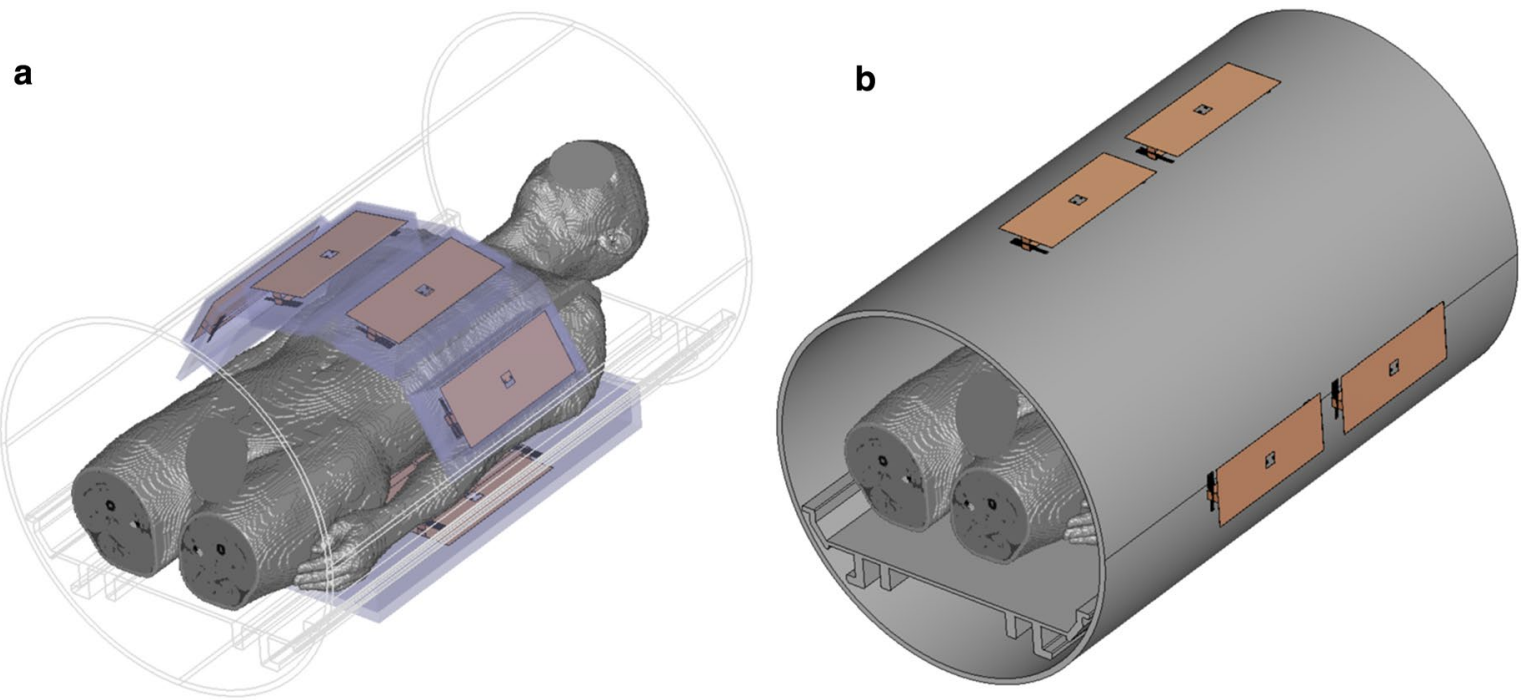

Fig. 1 Coil models used in this study. a Shows the flexible local body array and $\mathbf{b}$ shows the remote array. The simulation models contain the housing of the coil (where applicable), the patient table, the bore liner, and the magnet cryostat (not shown) 
MR environment (patient table, bore liner, gradient coil, and cryostat) as well as the coil housing where applicable.

The local array was tuned to resonance and matched to $50 \mathrm{Ohms}$ using a capacitor network in a co-simulation. The $2 \times 4$ remote array configuration was ideally tuned and decoupled by applying a decoupling matrix consisting of lossless inductors and capacitors that interconnects the transmit elements $[25,26]$. No decoupling matrix was applied to the local array.

A heterogeneous body model (male, $174 \mathrm{~cm}, 70 \mathrm{~kg}$, tissue resolution $2 \times 2 \times 2 \mathrm{~mm}^{3}$ ) [27] in head-first supine position with the liver-kidney region in the center was used for both arrays. The simulation domain was discretized with approximately 65 million mesh cells. Matrices for $10 \mathrm{~g}$-averaged local SAR were calculated for both setups resulting in 7.6 million matrices for the local array and 6.5 million matrices for the remote array.

\section{Algorithm implementation and calculation of results}

The algorithms were implemented in Matlab (The Mathworks Inc., Natick, MA, USA) with a high degree of vectorization and other optimizations to speed up the calculations. The software developed for this paper is available as open source at sourceforge.net (https://sourceforge.net/projects/ relative-overestimation-vop/). All compressions were performed on a PC with two 6-core Xeon X5690 processors (Intel, Santa Clara, CA, USA) with $128 \mathrm{~GB}$ of $1333 \mathrm{MHz}$ DDR3 RAM.

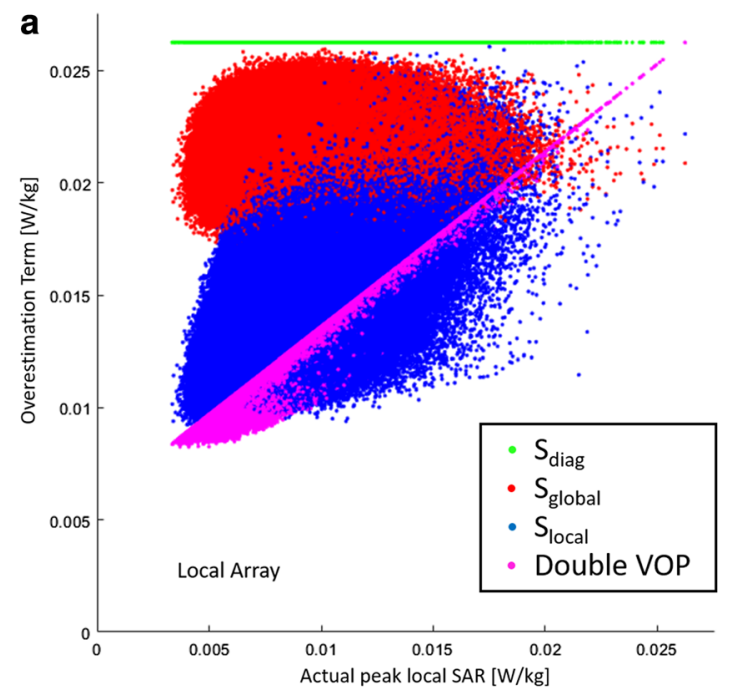

Fig. 2 Comparison of the value of the overestimation term for the different strategies for $\mathbf{a}$ the local array and $\mathbf{b}$ the remote array for a set of 1 million random excitation vectors with unit power. The $x$-axis shows the actual maximum local SAR calculated with the uncom-
1. The original dataset was compressed in the four ways explained above:

With the global SAR matrix ( $\left.S_{\text {global }}\right)$ according to Lee et al. [19] as the first reference standard

2. With a diagonal matrix ( $S_{\text {diag }}$ ) where all values on the main diagonal are equal to the worst-case local SAR, leading to an overestimation that is independent of actual local SAR as the second reference standard

3. With the new approach using a matrix approximating local SAR $\left(S_{\text {local }}\right)$

4. with the new approach utilizing a set of pre-calculated VOPs to define the maximum overestimation (Double VOP). The value of $\varepsilon_{\mathrm{G}, \text { pre }}$ was chosen to obtain 10 or fewer pre-calculated VOPs.

Furthermore, a compression with the Eichfelder algorithm was performed for comparison.

The compressions for all different strategies were repeated with different factors $\varepsilon_{\mathrm{G}}$ to obtain datasets with different numbers of VOPs. The SAR results obtained with these VOPs were then compared to the results of the uncompressed dataset for 1 million random excitation vectors to find the maximum relative overestimation.

\section{Results}

The pre-calculations for the Double VOP approach took less than $10 \mathrm{~min}$ and resulted in ten pre-calculated VOPs for the local array $\left(\varepsilon_{\mathrm{G} \text {,pre }}=0.2\right)$ and nine pre-calculated VOPs for the remote array $\left(\varepsilon_{\mathrm{G}, \text { pre }}=0.1\right)$.

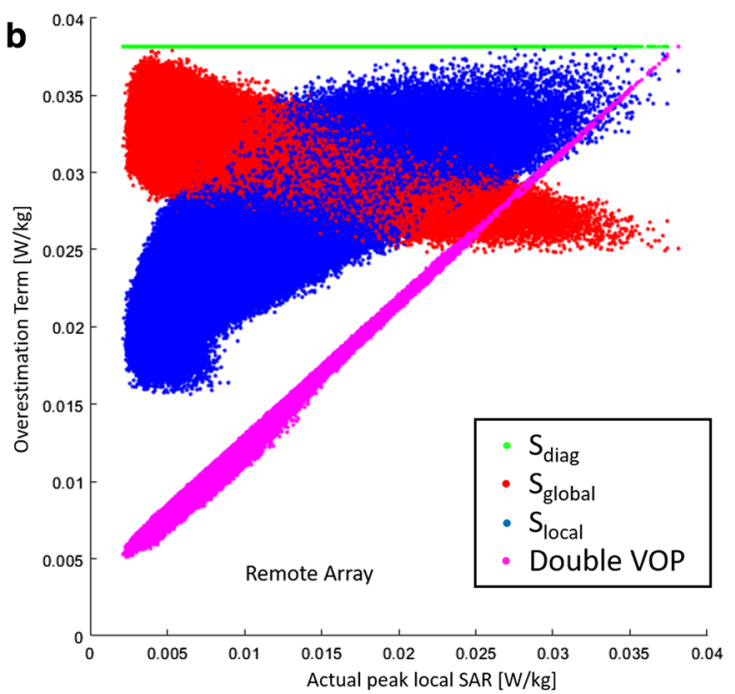

pressed set of SAR matrices; the $y$-axis shows the result of the overestimation term resulting from the respective excitation vector. Each overestimation term was normalized so that its maximum corresponds to worst-case actual local SAR 

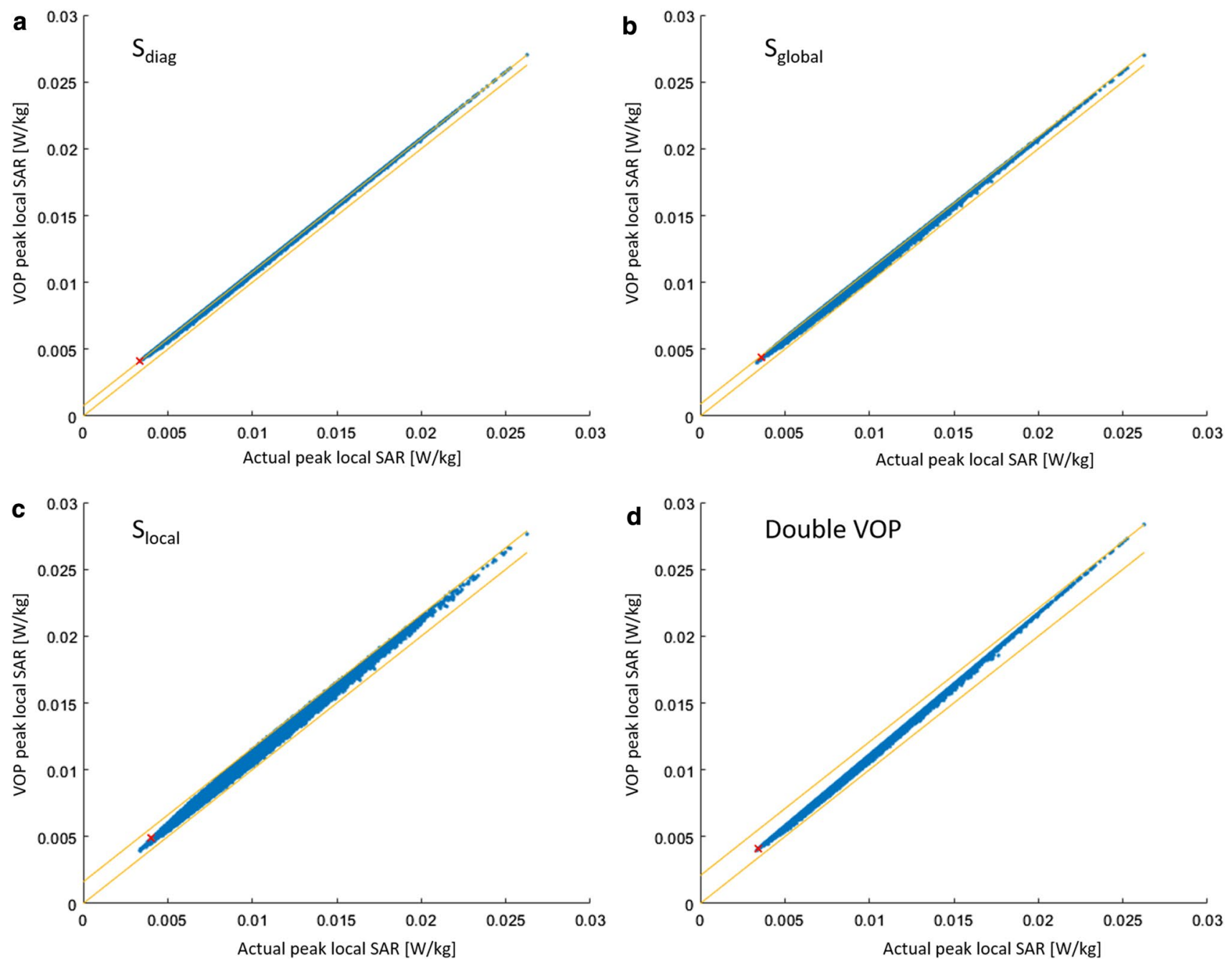

Fig. 3 Example results for the local array. The $x$-axis shows the actual local SAR calculated from the uncompressed dataset, while the $y$-axis shows the result of the respective compressed dataset with around 100 VOPs. A red cross marks the position of the maximum relative

The overestimation terms from Eqs. (1), (2), and (6) were compared to the actual SAR value calculated with the uncompressed dataset and 1 million random excitation vectors. For readability, the values were normalized so that their maximum values are equal to the worst-case actual local SAR for unit power excitation. The results for both array configurations are shown in Fig. 2. Figure 2a shows the results for the local array. While the results for $S_{\text {diag }}$ and $S_{\text {global }}$ are more or less uncorrelated with the actual local SAR, the overestimation terms for $S_{\text {local }}$ and especially Double VOP are lower when actual local SAR is lower.

In the case of the remote array, there is a visible negative correlation between the overestimation term of $S_{\text {global }}$ and the actual SAR (Fig. 2b). For low actual SAR values, the overestimation term is larger than it is for higher local SAR values, leading to a potentially higher relative overestimation

overestimation in each figure. The yellow lines denote the upper and lower bound (maximum absolute overestimation and zero overestimation). Note that for Double VOP and $S_{\text {local }}$, the values of VOP SAR adapt to the lower bound for low actual SAR values

at low SAR values. The overestimation terms for $S_{\text {local }}$ and Double VOP show a visible positive correlation with actual local SAR, resulting in smaller relative overestimation at low SAR values.

Example results in Fig. 3 show the VOP-calculated maximum local SAR versus the actual maximum local SAR in the local coil configuration for $S_{\text {diag }}$ (a), $S_{\text {global }}\left(\right.$ b), $S_{\text {local }}$ (c), and Double VOP (d), while Fig. 4 shows the absolute overestimation. The number of VOPs for all cases was approximately 100 , corresponding to a minimum overestimation term of around $2 \%$ of the worst case local SAR. Red crosses denote the position of the maximum relative overestimation; the yellow line is the unity line, included for reference. First, it can be noted that no underestimation occurred, which was to be expected since the mathematical concept has already been proved to be correct by Lee et al. [19]. It can be seen 

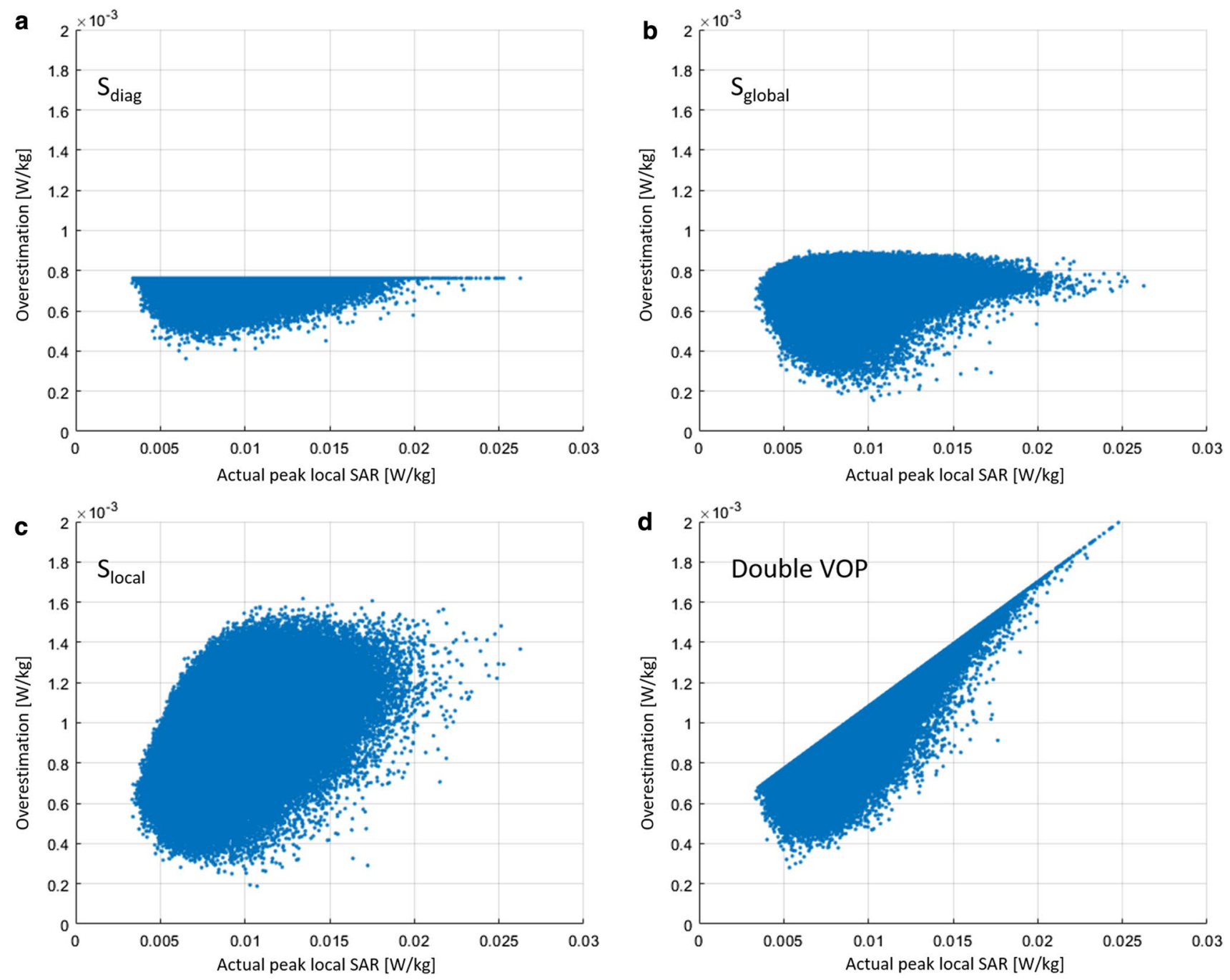

Fig. 4 Absolute overestimation of the VOP-calculated SAR versus the actual SAR for the local array. Each respective set contains a total of around 100 VOPs

that the four different overestimation terms result in different actual overestimations. Using $S_{\text {diag }}$ the maximum absolute overestimation for a given actual SAR value is constant versus the actual SAR, which can also be appreciated in Fig. 4a, while in the other three cases it shows some dependence on the actual local SAR. $S_{\text {local }}$ shows a reduction in the absolute overestimation below approximately $0.0075 \mathrm{~W} / \mathrm{kg}$ (Fig. 4c), while Double VOP (Fig. 4d) shows a linear behavior as could be expected from the results shown in Fig. 2a. The calculation time for the cases with a single matrix for the overestimation was approximately $90 \mathrm{~min}$ each, while the double VOP approach took $110 \mathrm{~min}$. For comparison, the Eichfelder algorithm needed $12 \mathrm{~min}$ for the 100 VOPs, while it took $55 \mathrm{~min}$ for the overestimation of $2 \%$ of worstcase SAR and produced 856 VOPs.

Example results for the remote array are given in Fig. 5. Again, the VOP-calculated maximum local SAR versus the actual local SAR in the local coil configuration for $S_{\text {diag }}$ (a), $S_{\text {global }}(\mathrm{b}), S_{\text {local }}(\mathrm{c})$, and Double VOP (d) is shown, while Fig. 6 provides the corresponding absolute overestimation. Here, the number of VOPs was around 45 for all cases, corresponding to a minimum overestimation term of around $2 \%$ of the worst-case local SAR. It can be noted that the ratio of the maximum actual SAR over the minimum actual SAR is significantly larger than for the case of the local array. The absolute overestimation using $S_{\text {global }}$ is larger for small actual SAR values than for large SAR values, significantly increasing the relative overestimation for small SAR values. Using $S_{\text {local }}$ and Double VOP, a smaller absolute overestimation occurs at lower actual SAR values. The calculation time for the cases with a single matrix for the overestimation was approximately $50 \mathrm{~min}$ each, while the double VOP approach took $70 \mathrm{~min}$. For comparison, the Eichfelder algorithm needed $12 \mathrm{~min}$ for the 45 VOPs, while 

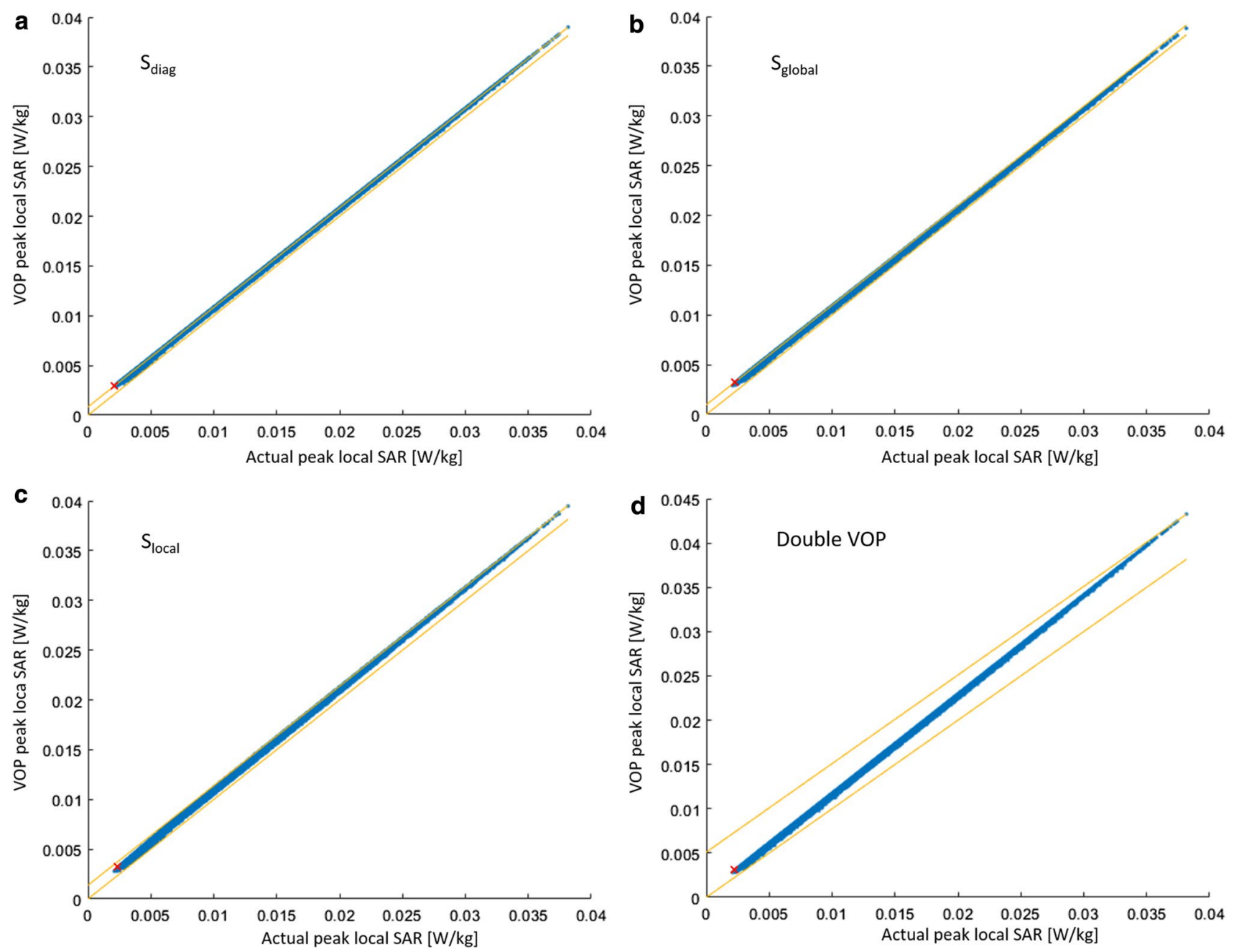

Fig. 5 Example results for the remote array. The $\mathrm{x}$-axis shows the actual local SAR calculated from the uncompressed dataset, while the $y$-axis shows the result of the respective compressed dataset with around 45 VOPs. A red cross marks the position of the maximum rel-

it took $55 \mathrm{~min}$ for the overestimation of $2 \%$ of worst-case SAR and produced 330 VOPs.

A direct comparison for all compression strategies is shown in Fig. 7. The figure shows the maximum relative overestimation in percent for the local array (7a) and remote array ( $7 b)$. Crosses denote the results obtained from compression; the connecting lines are included to improve readability. Note that for the Double VOP strategy, the number of VOPs is the number of VOPs from the compression plus the number of pre-calculated VOPs. As is to be expected, a larger number of VOPs results in a smaller maximum relative overestimation. In both configurations, Double VOP offers the best result, while $S_{\text {local }}$ outperforms $S_{\text {global }}$ in the remote array configuration but provides equivalent results in the local array configuration. In the remote array configuration, $S_{\text {global }}$ provides the overall worst result, being outperformed even by the simple $S_{\text {diag. }}$.

ative overestimation in each figure. The yellow lines denote the upper and lower bound (maximum absolute overestimation and zero overestimation). Note that for Double VOP and $S_{\text {local }}$, the values of VOP SAR adapt to the lower bound for low actual SAR values

In the areas between the dashed black lines in Fig. 7, the mean reduction in the number of VOPs is $17 \%$ for the local array and $24.6 \%$ for the remote array, respectively.

\section{Discussion}

In this work we show results with up to 180 VOPs for the Lee algorithm. This is close to the maximum number of 200 VOPs provided by Jin et al. as the maximum number that the pulse supervision of their vendor provided system can handle [28]. Due to the difference in compression efficiency, this number corresponds to roughly 1000 VOPs calculated by the Eichfelder algorithm when applying the same overestimation. The maximum number of VOPs that can be handled by the supervision system will depend on the 

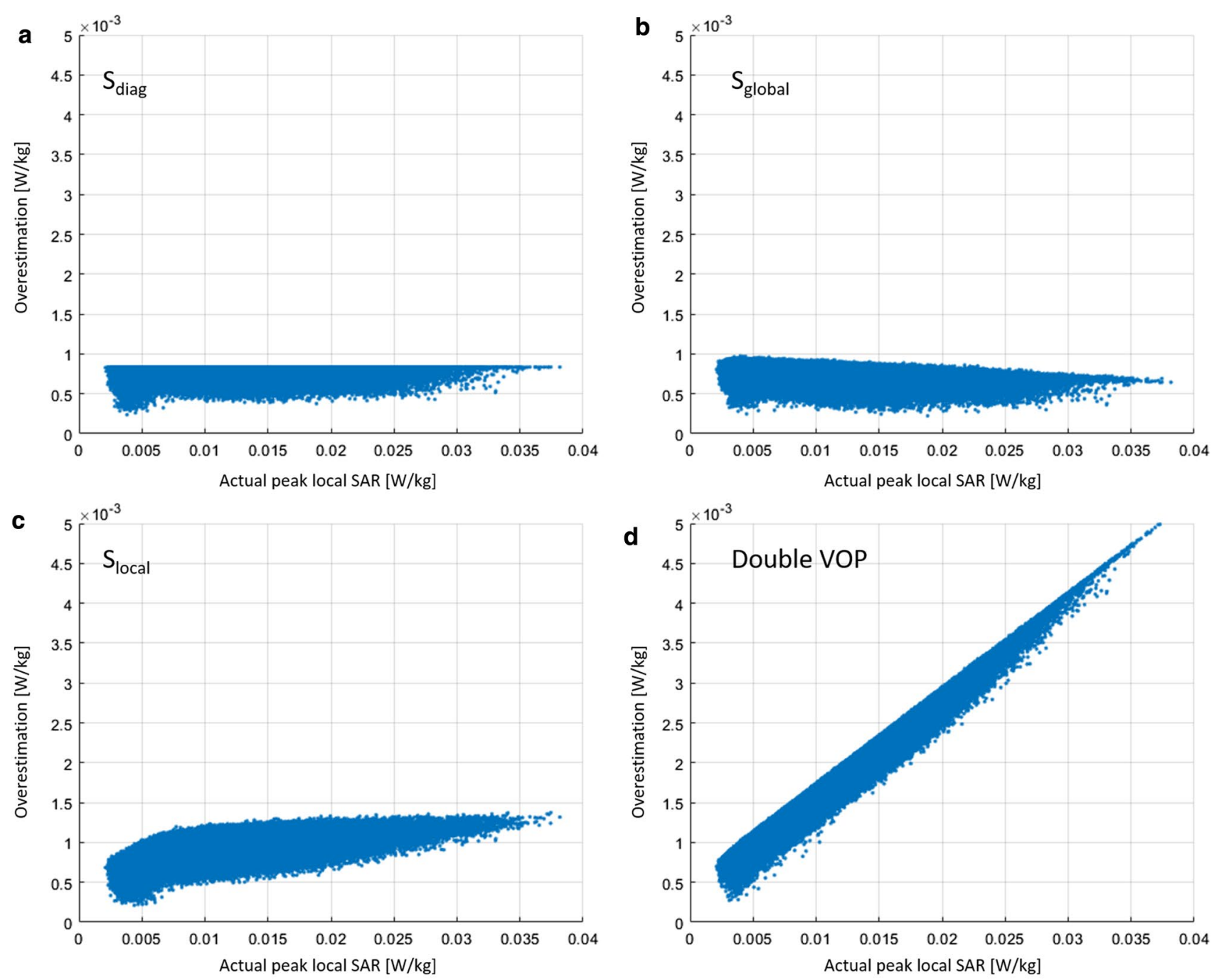

Fig. 6 Absolute overestimation of the VOP-calculated SAR versus the actual SAR for the remote array. Each respective set contains a total of around 45 VOPs

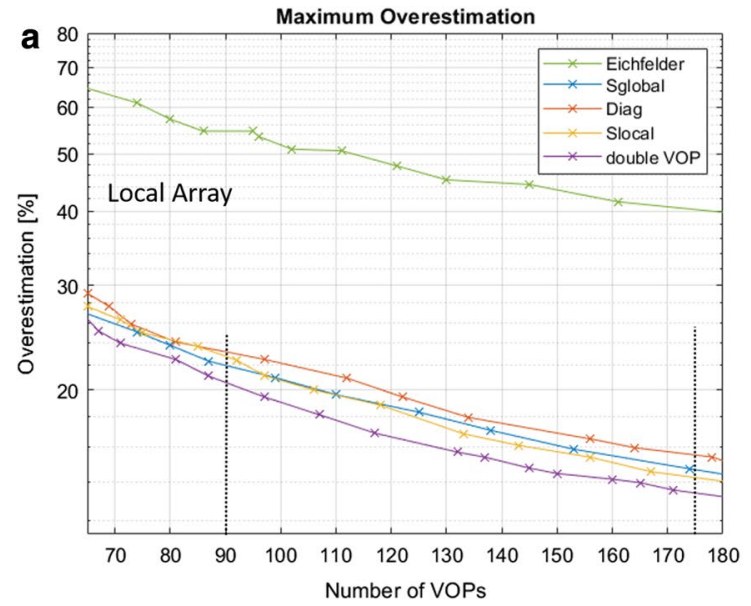

Fig. 7 Maximum relative overestimation versus number of VOPs. The crosses denote the calculated results; the connecting lines are included for better readability. Logarithmic scaling is used to include the results for the algorithm proposed by Eichfelder et al. In the areas between

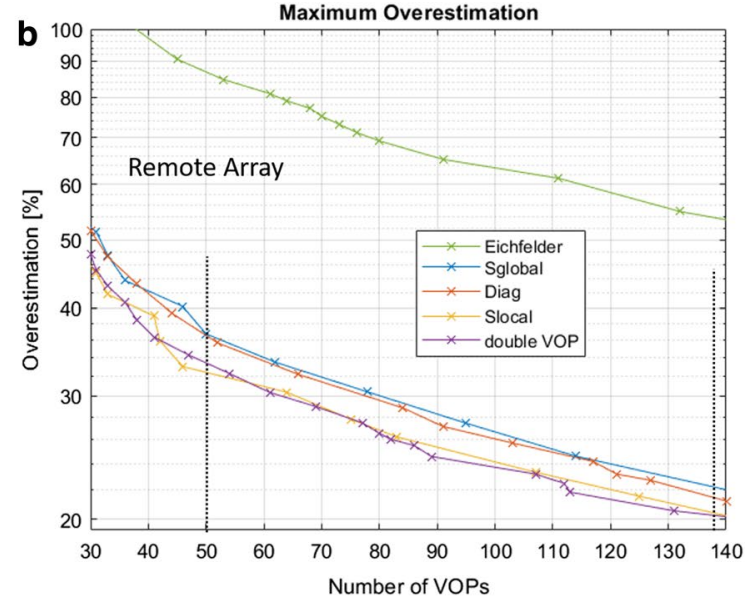

the dashed black lines, the mean reduction in the number of VOPs is $17 \%$ for the local array and $24.6 \%$ for the remote array, respectively 
particular hardware and software implementation as well on the number of elements in the transmit array.

The results for the overestimation terms show that $S_{\text {global }}$ is not necessarily a good choice. As shown here for the remote array, there are cases in which $S_{\text {global }}$ leads to an increased absolute overestimation at lower actual SAR values, leading to a disproportionately high relative overestimation. In this case, even a fixed overestimation $\left(S_{\text {diag }}\right)$ is preferable. Using an overestimation term that approximates actual local SAR $\left(S_{\text {local }}\right)$ proved preferable in this work, since it was at least as good as using $S_{\text {global }}$. The $S_{\text {local }}$ approach does not require any changes to the pulse calculation or pulse supervision software.

The overall best results for maximum relative overestimation were obtained using the Double VOP approach. A downside of this approach is the fact that the set of VOPs after compression is not directly compatible with previous algorithms for pulse calculation or supervision, since the results for two separate sets of VOPs have to be calculated and subsequently added up. Therefore, using this approach necessitates some software adaptations. While the implementation in many pulse calculation algorithms might be tricky because of having to implement the maximum of the VOP set used to calculate the overestimation term, the implementation into SAR supervision is straightforward. The calculation effort when calculating $N_{\text {VOPs }}$ VOPs in addition to $N_{\text {VOPs,pre }}$ pre-calculated VOPs should be almost the same as using the same number VOPs in the standard algorithm: $N_{\text {VOPs,standard }}=N_{\text {VOPs }}+N_{\text {VOPs,pre }}$. In this case, only $N_{\text {VOPs }}$ more addition operations are necessary in comparison to the standard method, which is not much effort considering that calculating the SAR for a single VOP in an eightchannel configuration takes 63 addition operations and 72 multiplication operations.

Using the proposed strategies $S_{\text {local }}$ and Double VOP, the number of VOPs could be reduced by around $20 \%$ while obtaining the same maximum relative overestimation in comparison to the original Lee algorithm. Overall, the two presented strategies provide a trade-off between maximum relative overestimation and maximum absolute overestimation.

\section{Conclusion}

In this paper, we present two strategies to reduce maximum relative overestimation in VOP compression. Both strategies are able to reduce the maximum relative overestimation for a fixed number of VOPs. Furthermore, we show that using global SAR for the overestimation term is not necessarily a good choice. Using the strategies proposed in this paper can lead to enhanced performance of VOP compressions and therefore more exact and/or faster calculation of local SAR. Practically, this implies improved performance of multichannel RF arrays in pTx applications.

Author contributions SO: study conception and design, acquisition of data, analysis and interpretation of data, drafting of manuscript. TMF: acquisition of data, critical revision. AKB: critical revision. MEL: critical revision. HHQ: critical revision

Funding Open Access funding enabled and organized by Projekt DEAL.

\section{Compliance with ethical standards}

Conflict of interest Stephan Orzada declares that he has no conflict of interest. Thomas M. Fiedler declares that he has no conflict of interest. Andreas K. Bitz declares that he has no conflict of interest. Mark E. Ladd declares that he has no conflict of interest. Harald H. Quick declares that he has no conflict of interest.

Ethical approval This article does not contain any studies with human participants or animals performed by any of the authors.

Open Access This article is licensed under a Creative Commons Attribution 4.0 International License, which permits use, sharing, adaptation, distribution and reproduction in any medium or format, as long as you give appropriate credit to the original author(s) and the source, provide a link to the Creative Commons licence, and indicate if changes were made. The images or other third party material in this article are included in the article's Creative Commons licence, unless indicated otherwise in a credit line to the material. If material is not included in the article's Creative Commons licence and your intended use is not permitted by statutory regulation or exceeds the permitted use, you will need to obtain permission directly from the copyright holder. To view a copy of this licence, visit http://creativecommons.org/licenses/by/4.0/.

\section{References}

1. Hoult DI, Phil D (2000) Sensitivity and power deposition in a high-field imaging experiment. J Magn Reson Imaging 12(1):46-67

2. Van de Moortele PF, Akgun C, Adriany G, Moeller S, Ritter J, Collins CM, Smith MB, Vaughan JT, Ugurbil K (2005) B(1) destructive interferences and spatial phase patterns at $7 \mathrm{~T}$ with a head transceiver array coil. Magn Reson Med 54(6):1503-1518

3. Deniz CM (2019) Parallel transmission for ultrahigh field MRI. Top Magn Reson Imaging 28(3):159-171

4. Padormo F, Beqiri A, Hajnal JV, Malik SJ (2016) Parallel transmission for ultrahigh-field imaging. NMR Biomed 29(9):1145-1161

5. Collins CM, Liu W, Swift BJ, Smith MB (2005) Combination of optimized transmit arrays and some receive array reconstruction methods can yield homogeneous images at very high frequencies. Magn Reson Med 54(6):1327-1332

6. Metzger GJ, Snyder C, Akgun C, Vaughan T, Ugurbil K, Van de Moortele PF (2008) Local B1+ shimming for prostate imaging with transceiver arrays at $7 \mathrm{~T}$ based on subject-dependent transmit phase measurements. Magn Reson Med 59(2):396-409 
7. Cloos MA, Boulant N, Luong M, Ferrand G, Giacomini E, Le Bihan D, Amadon A (2012) kT -points: short three-dimensional tailored RF pulses for flip-angle homogenization over an extended volume. Magn Reson Med 67(1):72-80

8. Setsompop K, Alagappan V, Gagoski B, Witzel T, Polimeni J, Potthast A, Hebrank F, Fontius U, Schmitt F, Wald LL, Adalsteinsson E (2008) Slice-selective RF pulses for in vivo B1+ inhomogeneity mitigation at 7 tesla using parallel RF excitation with a 16-element coil. Magn Reson Med 60(6):1422-1432

9. Saekho S, Yip CY, Noll DC, Boada FE, Stenger VA (2006) Fast$\mathrm{kz}$ three-dimensional tailored radiofrequency pulse for reduced B1 inhomogeneity. Magn Reson Med 55(4):719-724

10. Grissom W, Yip CY, Zhang Z, Stenger VA, Fessler JA, Noll DC (2006) Spatial domain method for the design of RF pulses in multicoil parallel excitation. Magn Reson Med 56(3):620-629

11. Katscher U, Bornert P, Leussler C, van den Brink JS (2003) Transmit SENSE. Magn Reson Med 49(1):144-150

12. Orzada S, Maderwald S, Poser BA, Bitz AK, Quick HH, Ladd ME (2010) RF excitation using time interleaved acquisition of modes (TIAMO) to address B1 inhomogeneity in high-field MRI. Magn Reson Med 64(2):327-333

13. International Electrotechnical Commission (IEC) (2015) IEC 60601-2-33 Medical electrical equipment-part 2-33: particular requirements for the basic safety and essential performance. Edition 3.2

14. Fiedler TM, Ladd ME, Bitz AK (2018) SAR simulations and safety. NeuroImage 168:33-58

15. Graesslin I, Vernickel P, Bornert P, Nehrke K, Mens G, Harvey P, Katscher U (2014) Comprehensive RF safety concept for parallel transmission MR. Magn Reson Med. https://doi. org $/ 10.1002 / \mathrm{mrm} .25425$

16. Gumbrecht R, Fontius U, Adolf H, Benner T, Schmitt F, Adalsteinsson E, Wald LL, Fautz H-P (2013) Online local SAR supervision for transmit arrays at 7 T. Proc Int Soc Mag Reson Med 21:4420

17. Zhu Y, Alon L, Deniz CM, Brown R, Sodickson DK (2012) System and SAR characterization in parallel RF transmission. Magn Reson Med 67(5):1367-1378

18. Eichfelder G, Gebhardt M (2011) Local specific absorption rate control for parallel transmission by virtual observation points. Magn Reson Med 66(5):1468-1476
19. Lee J, Gebhardt M, Wald LL, Adalsteinsson E (2012) Local SAR in parallel transmission pulse design. Magn Reson Med 67(6):1566-1578

20. Deniz CM, Vaidya MV, Sodickson DK, Lattanzi R (2016) Radiofrequency energy deposition and radiofrequency power requirements in parallel transmission with increasing distance from the coil to the sample. Magn Reson Med 75(1):423-432

21. Strang G (2009) Introduction to linear algebra, 9th edn. WellesleyCambridge Press, Wellesley

22. Zhan X (2002) Matrix inequalities, 6th edn. Springer, Berlin

23. Rietsch SHG, Quick HH, Orzada S (2015) Impact of different meander sizes on the RF transmit performance and coupling of microstrip line elements at $7 \mathrm{~T}$. Med Phys 42(8):4542-4552

24. Orzada S, Quick HH, Ladd ME, Bahr A, Bolz T, Yazdanbakhsh P, Solbach K, Bitz AK (2009) A flexible 8-channel transmit/receive body coil for $7 \mathrm{~T}$ human imaging. In: Proceedings of the 17th scientific meeting, international society for magnetic resonance in medicine, Honolulu, p 2999.

25. Mahmood Z, Guerin B, Adalsteinsson E, Wald LL, Daniel L (2013) An automated framework to decouple pTx arrays with many channels. Proc Int Soc Magn Reson Med 21:2722

26. Mahmood Z, Guerin B, Adalsteinsson E, Wald LL, Daniel L (2014) Design of a robust decoupling matrix for high field parallel transmit arrays. Proc Intl Soc Mag Reson Med 22:548

27. Christ A, Kainz W, Hahn EG, Honegger K, Zefferer M, Neufeld E, Rascher W, Janka R, Bautz W, Chen J, Kiefer B, Schmitt P, Hollenbach HP, Shen J, Oberle M, Szczerba D, Kam A, Guag JW, Kuster N (2010) The virtual family-development of surface-based anatomical models of two adults and two children for dosimetric simulations. Phys Med Biol 55(2):N23-38

28. Jin J, Weber E, Destruel A, O'Brien K, Henin B, Engstrom C, Crozier S (2018) An open 8-channel parallel transmission coil for static and dynamic 7T MRI of the knee and ankle joints at multiple postures. Magn Reson Med 79(3):1804-1816

Publisher's Note Springer Nature remains neutral with regard to jurisdictional claims in published maps and institutional affiliations. 